\title{
Vorwort zur deutschsprachigen Ausgabe
}

Die zweite amerikanische Auflage des inzwischen klassisch gewordenen "Jackson" als eine der modernsten und umfassendsten Darstellungen der Klassischen Elektrodynamik zu bezeichnen, erscheint kaum übertrieben. Enthalten doch nur wenige vergleichbare Bücher so viele Brückenschläge zur Atom-, und Kernphysik und geben einen so bestechenden Uberblick über die gesamte Elektrodynamik. Eine deutsche Ubersetzung dieses Werkes empfahl sich auch deswegen, weil die in ihm behandelten Zusammenhänge oftmals schon in der deutschsprachigen Formulierung dem Leser - und ganz besonders dem Studenten und Anfänger - begriffliche Schwierigkeiten bereiten können.

Der Text wurde so frei wie möglich übersetzt, ohne jedoch das Original $z u$ verfalschen. Umgestaltet wurde lediglich der Abschnitt über die Delta-Funktion (S. $39-40$ ). Vom Ubersetzer hinzugefügte Fußnoten wurden durch ein Kreuz ${ }^{+}$) gekennzeichnet. Zusätze und Änderungen wurden auch in den Literaturhinweisen $b z w$. in der Bibliographie vorgenommen. Wo immer von einem im Original angegebenen Titel eine deutsche Ubersetzung existiert, wurde diese angegeben (mit einem entsprechenden Ujbersetzungsvermerk). Ferner wurden einige zusatzliche deutsche Titel in die Literaturhinweise aufgenommen (und durch einen stern gekennzeichnet), um dem deutschen Leser die Vertiefung des Stoffes $z u$ erleichtern.

Um die Herstellungskosten zu senken, wurden das Maschinenskript der tubersetzung und sämtiche Formeln und symbole des Originalbuches für die Reproduktion verwendet.

Berlin, im Herbst 1980

$$
\text { K. Müller }
$$


